\title{
Tangence
}

\section{À la croisée du hasard et du déterminisme : atomisme logique et fractalité postmoderne dans La démarche du crabe de Monique LaRue}

\section{Lucie Guillemette}

Numéro 61, décembre 1999

Savoir et littérature

URI : https://id.erudit.org/iderudit/008168ar

DOI : https://doi.org/10.7202/008168ar

Aller au sommaire du numéro

Éditeur(s)

Presses de l'Université du Québec

ISSN

0226-9554 (imprimé)

1710-0305 (numérique)

Découvrir la revue

Citer cet article

Guillemette, L. (1999). À la croisée du hasard et du déterminisme : atomisme

logique et fractalité postmoderne dans La démarche du crabe de Monique

LaRue. Tangence, (61), 113-146. https://doi.org/10.7202/008168ar 


\section{À la croisée du hasard et du déterminisme : atomisme logique et fractalité postmoderne dans La démarche du crabe de Monique LaRue}

\section{Lucie Guillemette, Université du Québec à Trois-Rivières}

Si les questions liées aux principes fondateurs de l'homme et à la formation du monde sont exposées d'un même élan dans les premiers écrits de la philosophie, elles retiennent également l'attention de nombreux écrivains de la postmodernité ${ }^{1}$. Dans le prolongement de la pensée antique, "la démarche scientifique est réintégrée à la culture " 2 , alors que bien des auteurs renouent le dialogue entre science et philosophie. Certes, le rôle assigné à la faculté de penser a évolué depuis vingt-cinq siècles. À l'époque de l'Antiquité grecque, faut-il le rappeler, le "connais-toi toimême" cher à Socrate rend compte de la foi en la raison par laquelle l'homme peut atteindre à la connaissance de soi. Mais à l'ère d'un postmodernisme en faveur duquel la raison ne peut plus opérer selon un modèle unique, le savoir comporte un aspect spéculatif alors que l'identité avec ses composantes illusoires de permanence et de continuité se pose en $\operatorname{artefact}^{3}$. Tout se passe comme si "un réel voilé, incertain, sans nécessité" était devenu le trait majeur de la science et de l'épistémologie contemporaines ${ }^{4}$. Ainsi perçu comme un univers mouvant, propice au

1. Il convient d'apporter quelques précisions à la notion "postmoderne" qui reste encore floue et controversée. Dans le contexte de notre étude, la postmodernité est décrite d'abord comme un courant de pensée "prenant ses distances par rapport à toute revendication de la Raison, de l'Absolu, de la Vérité". Voir Gilbert Hottois, De la Renaissance à la Postmodernité. Une histoire de la philosophie moderne et contemporaine, Paris et Bruxelles, De Boeck \& Larcier, coll. "Le point philosophique", 1998, p. 446.

2. Jacqueline Russ, La marche des idées contemporaines. Un panorama de la modernité, Paris, Armand Colin, 1994, p. 189.

3. "L'absence d'identité bien définie et de consistance" constitue l'une des principales critiques que l'on formule à l'endroit du courant de pensée postmoderne. Voir Gilbert Hottois, op. cit., p. 446.

4. C'est l'une des thèses que défend Bernard d'Espagnat: "Si le réel en soi refuse de nous dire ce qu'il est — ou comment il est — du moins consent-il 
désordre et à la confusion, le monde se présente comme un lieu complexe où l'indétermination et l'aléatoire se sont substitués aux certitudes héritées des principes du rationalisme dont le but ultime, depuis Descartes, a été de dominer la nature ${ }^{5}$. Pareilles interrogations relatives à la modernité rationaliste et au modèle de la pensée unifiante que ce courant revendique ont traversé les âges pour se retrouver en cette fin de siècle au cour des textes littéraires, sous les formes les plus diverses. À l'aube du troisième millénaire, plusieurs écrivains "incrédules à l'endroit des métarécits " 6 abordent le fait identitaire sous un angle philosophique particulier. Ils mettent en question le pouvoir et les vertus de la digne et docte raison dont le progressisme des Lumières a fait l'apologie. Dans les fictions contemporaines, nombreux sont les personnages romanesques à la recherche d'un sens au sein d'un monde pour lequel les critères de vérité et d'objectivité ne tiennent plus. C'est à tout le moins ce qui se dégage des textes de facture postmoderne où l'actualisation d'un discours sur l'identité, désormais comprise comme équivoque et relative, va de pair avec "l'abandon des grands récits de légitimation de la civilisation occidentale [...] qui tous prétendaient conduire l'humanité vers un salut unique et assuré " 7 . À l'heure où les récits fondateurs aux visées homogénéisantes et totalisantes se sont effrités, le discours

à nous faire savoir, dans une certaine mesure, ce qu'il n'est pas". Une incertaine réalité. Le monde quantique, la connaissance et la durée, Paris, Bordas, 1985 , p. 269.

5. "L'universalisme rationaliste, la foi dans la science et la technique, la domination-exploitation de la nature par et pour l'humanité, la foi dans la prise en charge de l'humanité par elle-même, le mépris du passé, l'utopisme", autant de traits qui s'nscrivent au cour du mythe de la modernité auquel s'oppose par définition le postmoderne. Gilbert Hottois, op. cit., p. 445. Avec l'absence d'une logique universellement reconnue, la science contemporaine cesse de légiférer dans l'absolu et souligne les limites de toute formalisation. Le domaine scientifique témoigne d'une fin des certitudes, un crépuscule des évidences. Dans la même foulée, Ilya Prigogine et Isabelle Stenghers décrivent la connaissance scientifique comme un lieu où nulle stabilité n'est plus garantie. Voir La nouvelle alliance. Métamorphose de la science, Paris, Gallimard, 1979.

6. L'expression est empruntée à Jean-François Lyotard. Il s'agit d'une incrédulité à l'endroit des récits fondateurs. Pensons à l'histoire judéo-chrétienne, l'hégélianisme, le positivisme, l'évolutionnisme, le socialisme et le marxisme, etc. Voir La condition postmoderne. Rapport sur le savoir, Paris, Éditions de Minuit, 1979. Jacqueline Russ décrit pour sa part cette fin de siècle comme "la fin de la raison triomphante". Op. cit., p. 6.

7. Gilbert Hottois, op. cit., p. 445. 
littéraire associe l'identité à une donnée floue et inconsistante qui échappe au raisonnement logique, démonstratif et normatif. Il résulte d'un tel changement de paradigme ${ }^{8}$ un relativisme qui tend à dissoudre les signifiés figés du Même et de l'Autre, comme en témoignent les recherches de pointe en épistémologie.

À l'exemple des auteurs orientés vers des problématiques postmodernes, Monique LaRue s'intéresse à la question de l'identité dans un contexte d'écriture marqué par la multiplicité d'un monde en devenir, à travers lequel réalité et fiction ne tendent plus à s'opposer. À certains égards, l'œuvre romanesque de LaRue consiste à dégager de diverses traditions de pensée des axiomes qui n'accordent plus à la raison et à l'essence du langage une importance privilégiée pour dire et expliquer le monde? . En 1989 paraît Copies conformes ${ }^{10}$ dont le récit confond sans ambages le vrai et le faux au sein d'une Californie figurant comme un lieu éminent de découvertes issues de la technologie informatique. Rappelons que la fiction s'articule autour de la notion de simulacre dont Lucrèce a livré une théorie pénétrante dans son poème De la nature des choses. À partir d'une interprétation contemporaine du matérialisme antique de Lucrèce, disciple et continuateur des travaux d'Épicure, la romancière façonne le matériau textuel à même le langage des apparences et de l'imitation. Le texte récuse ainsi le monde idéel platonicien correspondant aux essences immatérielles et immuables. Or, Monique LaRue introduit des thèses philosophiques du même type dans un plus récent roman paru en 1995, La démarche du crabe $^{11}$. Toujours dans la filiation du matérialisme, l'ouvrage fait de la question des origines, inextricablement liée à la genèse des êtres qui naissent puis qui meurent, la thématique centrale de la diégèse. Si l'écrivaine répudie à nouveau le réalisme platonicien au fil de l'élaboration de la trame narrative, son propos prend appui sur les travaux de certains philosophes de la nature suivant lesquels l'univers est corruptible et ne se constitue d'autre substance que la matière. C'est plus particulièrement en se référant à

\footnotetext{
8. Il s'agit du paradigme de la complexité.

9. À toutes fins utiles, signalons que la romancière Monique LaRue est diplômée de philosophie.

10. Monique LaRue, Copies conformes, Montréal et Paris, Lacombe et Denoël, 1989, $190 \mathrm{p}$.

11. Id., La démarche du crabe, Montréal, Boréal, 1995, 221 p. Toute référence ultérieure à ce texte sera indiquée par un chiffre placé entre parenthèses.
} 
116

la doctrine de l'atomisme, selon laquelle l'univers est formé d'atomes associés en combinaisons fortuites, que l'auteure développe la structure romanesque. Une telle pratique discursive donne lieu notamment à une réflexion sur l'existence des êtres et des choses. Or, cette même réflexion s'écarte du cadre de la logique classique dominée par une pensée logocentrique. Afin de cerner la dynamique du récit qui nous occupe, il convient de s'attarder à la signification de l'intertexte à orientation philosophique dans La démarche du crabe. Notre questionnement s'énonce ainsi : suivant quels procédés textuels de l'autoreprésentation, l'instance narrative entreprend-elle de décrire le passé afin d'en extraire un sens quelconque; à quelles fins le récit littéraire transpose-t-il un énoncé théorique reprenant certaines thèses atomistes relatives à la compréhension de l'Univers; comment prend forme une pensée relativiste et postmoderne qui situe un discours de l'identité à la croisée du déterminisme et du hasard au sein de la narration. Autant d'interrogations qui se greffent à la présente étude consacrée à la problématique identitaire prévalant dans un roman postmoderne aux allures philosophiques.

Ce triple questionnement s'amorce avec la description et l'analyse de l'autoreprésentation textuelle mise en rapport avec des contenus philosophiques au sein du roman de LaRue. Pour utiliser la terminologie de Gérard Genette, il s'agit d'examiner la "transtextualité" 12 . De l'étude du procédé se dégage un discours philosophique portant sur la problématique de l'identité et son heuristique. La lecture des savoirs ${ }^{13}$ à laquelle nous procédons consiste à conjuguer un réseau de connaissances transtextuelles spécifiques à une pratique énonciative qui traduit la mouvance d'un je scripteur et agonisant. Selon nos hypothèses, l'acte d'énonciation rend possible une dynamique identitaire caracté-

12. Nous utilisons la notion de transtextualité dans le sens où l'entend Gérard Genette. Décrite comme l'objet même de la poétique, la transcendance textuelle rend compte du système de relations qu'un texte établit avec d'autres textes. Voir Palimpsestes. La littérature au second degré, Paris, Seuil, 1982, p. 7.

13. Comme l'indique Michel Pierssens, "un savoir, dès lors qu'il devient texte, quand la parole le traduit, ne peut être [...] qu'un hybride issu d'une généalogie compliquée. Aussi faut-il quand il s'agit d'en comprendre les effets en littérature en parler au pluriel : c'est à des savoirs que nous avons à faire, plutôt qu'au Savoir universel et majuscule". Michel Pierssens, Savoirs à l'ouvre. Essais d'épistémocritique, Lille, Presses universitaires de Lille, 1990, p. 8 . 
risée par un trajet oblique et discontinu, relativisé par les marques oppositionnelles de la continuité et du changement apposées à l'une ou à l'autre conception de l'individuation exprimées dans l'énoncé. Plus précisément, le registre énonciatif prend en charge un parcours généalogique particulier, celui d'un homme qui, au rythme d'un processus d'écriture à partir duquel il s'autoreprésente, fait osciller le récit entre le hasard et le déterminisme, le contingent et le nécessaire. C'est donc la pratique autoréférentielle d'une subjectivité masculine qui deviendra l'objet de nos investigations. Voyons comment la mise en abyme d'un je scripteur s'accorde à un discours sur la nature au cour de laquelle gisent le hasard, l'aléatoire.

\section{Autoreprésentation textuelle et dérive de l'identité : l'altération spatio-temporelle de l'énonciation}

"La démarche du crabe", dont le texte tire son intitulé, est celle d'un Montréalais dans la quarantaine, qui exerce la profession de dentiste. Il s'agit de Luc-Azade Santerre vivant dans "une forteresse de parvenus" (14), "ces décors nouveau riche" de Ville de Mont-Royal (46). Signalons que l'abréviation anglaise est préférée au sein du discours narratif: TMR se réfère ainsi à Town of Mount Royal. Rongé par un cancer, la rate hypertrophiée, le personnage amorce un projet d'écriture depuis un hôpital montréalais posé comme l'ici de l'énonciation. Alors que son être se corrompt, le protagoniste entreprend de produire le récit de son odyssée spatio-temporelle. Le but est d'effectuer l'archéologie sentimentale de ses proches et de lui-même. L'histoire relatée dans les carnets du dentiste s'amorce au printemps de l'année 1987 et se termine en mai de l'année suivante. Cette structure chronologique laisse supposer que le temps de l'énonciation coïncide avec l'hospitalisation de Santerre au printemps de l'an 1988, laquelle est consécutive à un voyage de quelques jours à la campagne et en forêt. Cependant, le récit des douze derniers mois de l'existence du personnage est interrompu par une longue analepse consacrée à des événements s'étant déroulés durant la tenue de l'Exposition universelle de Montréal, soit du 30 avril au 31 octobre 1967. Le discours rétrospectif dont la portée est de vingt ans s'intitule "L'Exposition". Cette partie constitue le plus long chapitre du récit et entame la deuxième moitié du roman. Quatre chapitres précèdent le passage analeptique. Il s'agit de "Sarah", "Michelle", "Docteur Luc-Azade Santerre" et "La 
118

démarche du crabe". Le manuscrit se compose de trois dernières sections dont les intitulés respectifs se donnent à lire comme des syntagmes topographiques: "Rive sud", "Rive nord" et "La forêt". Mis en abyme, le récit est divisé en sept parties qui reproduisent à divers degrés le parcours généalogique d'un homme en quête de son passé.

C'est donc depuis un lieu évoquant la maladie et la mort que s'actualise le récit. Le narrateur reproduit alors les principaux événements, révélateurs de sa remontée dans le labyrinthe des souvenirs. Au sein d'un manuscrit qu'il définit lui-même comme un "testament" (69 et 103), Luc-Azade Santerre reconstitue son arbre généalogique en passant par la brève histoire anecdotique de ses parents et grands-parents. Pour ce faire, le citadin abandonne son cabinet et son épouse. Après maintes tergiversations, le protagoniste quitte Montréal et des lieux urbains familiers. Il se rend d'abord sur la rive sud qui épouse le relief du bas du fleuve Saint-Laurent, là où il allait en vacances avec sa mère lorsqu'il était enfant. Son périple se prolonge sur le littoral opposé, dans la région de Charlevoix; il y rencontre des gens qui lui parlent de sa grand-mère maternelle, demeurée jusque là une inconnue. Il termine son voyage au coeur d'une forêt qui s'avère un lieu mémoriel évocateur. Comme le suggère une lecture onomastique, le signifiant du nom propre "Santerre", transformé en son homophone "sans terre", évoque littéralement une absence d'origines et justifie à un premier niveau l'activité de déchiffrement auquel va procéder sans relâche le personnage.

Voyons d'entrée de jeu comment l'énonciation émanant de lieux macabres institue la quête identitaire. Le récit du dentiste Santerre est relaté sous la forme de la première personne. Le personnage qui raconte et se raconte se fait l'auteur d'un roman: "J'avais toujours voulu écrire un roman, et maintenant ce désir inassouvi me gonflait d'enthousiasme." (216). Le je narrant se pose comme un je scripteur, des plus déterminés à se dire: "J'ai commencé à ressasser une très ancienne frustration: j'aurais voulu écrire. J'aurais voulu faire des lettres, de la philosophie." (39) Santerre prétend "[qu'il a] toujours aimé faire de la philosophie comme on fait de la cuisine, de la chimie, du bricolage" (79). Autrement dit, l'activité de réflexion inhérente à l'écriture s'apparente à une tâche de tous les jours, n'exigeant aucun effort de la part du protagoniste. En fait, le je narrant confesse que son projet romanesque correspond à "une chère 
idée de jeunesse, une vocation inassouvie" (103). Il ajoute encore que "l'idée d'écrire faisait sa place en [lui] naturelle, comme elle est venue depuis toujours sans doute aux humains" (102). Bref, l'intérêt marqué que manifeste le professionnel envers les choses de l'esprit ne semble point suscité par une raison souveraine, organisatrice des pensées et des actions humaines. Au contraire, le projet d'écriture est identifié à un désir "très ancien" que la mémoire paraît avoir effacé. Alors que le rêve de jeunesse émerge de l'oubli, le narrateur agonisant s'inscrit dans un processus créateur où "[les mots créent] une réalité" (63).

Nul doute que la prise en charge de l'énonciation par un moi fictif se sachant condamné redéfinit la part du fictif et du non fictif dans un récit où les mots couchés sur le papier ont pour fonction de suspendre le temps: "La concentration de mon esprit sur les mots de la langue française et sur ce fil solide, souple invisible, qui arme les récits pour leur voyage dans le temps et dans l'espace, m'aura de toute façon évité de sentir et de vivre pendant quelques heures." (17) Se cramponnant aux signes matériels de l'écrit, Santerre entend échapper à la clôture téléologique du monde social en complétant la rédaction d'un ouvrage romanesque. Bien que le je narré ignore qu'il est atteint d'un mal incurable au moment de son errance sur les rives du SaintLaurent, le je narrant, depuis l'hôpital, traduit clairement à travers son manuscrit la présence de la mort qui le guette. Sur le plan de l'énonciation, cette distinction est engendrée par des déictiques qui déterminent les contextes spatio-temporels de l'hier et de l'aujourd'hui: "Maintenant, je comprends que mon récit est aussi celui de la mort avançant par paliers" (11); "comment ai-je pu éviter de voir que la mort elle-même me faisait signe?" (193) Le narrateur met l'accent sur le fait que le je autoreprésenté de son récit se pose comme différent du je scripteur. Tel qu'énoncé, l'écart entre les deux instances est fonction de l'expérience sensible et de la durée qu'implique la perception. Pendant les mois précédant son hospitalisation, le personnage est si absorbé par l'idée d'écrire qu'il ne perçoit pas la dégradation de sa condition physique: "Je n'ai pas vu à quel point j'avais maigri, je n'ai pas remarqué mon teint cendreux." (70) Loin de se douter de l'affection physique qui le mine, Santerre présume être victime de malaises psychologiques, ce en quoi il se trompe bien entendu. Incapable d'interpréter des signes cliniques pourtant apparents, le dentiste fait fi des symptômes exprimant une 
altération d'origine physique. On comprend alors pourquoi le je narrant multiplie les énoncés qui se réfèrent au savoir défaillant du je narré: "je ne comprenais pas" (87), "j'étais loin de me rendre compte" (31), "je ne pouvais pas savoir" (131), "je ne soupçonnais pas" (57). Dans le cas de Santerre, tout se passe comme si l'identité était relative au temps de l'écriture dont la prolifération de signifiants semble initier un processus de transformation infinie du je en un autre et vice versa: "Ces mots que je puis écrire maintenant, je n'aurais pu les expliquer alors, ni comprendre ce qu'ils pouvaient vouloir [sic] dire." (67) Une fois le diagnostic médical rendu, la démarche scripturale du protagoniste rattrape le temps de l'énonciation.

Lors du passage consacré au voyage qui a mené l'homme de la ville au fond des bois, le discours du personnage se modifie quelque peu, dans la mesure où celui-ci affirme avoir anticipé sa mort. Véritable mélange de souvenirs et d'imagination, le texte autoréférentiel se fait le reflet de la corruption d'un être dont la pensée est brouillée à cause de la maladie: "Maintenant que je suis parvenu au seuil, j'affirme que tout ce temps, j'ai deviné, sans la reconnaître cette noble compagne sécrétant sa bile noire, me voilant la vue, m'éloignant des miens, progressant en moi comme un crabe." (221) De cette aventure scripturale découle l'image du crabe qui renvoie au signe horoscopique du cancer, lui-même rendant compte d'une maladie pernicieuse. Chancelant, l'homme introduit au sein de ses carnets une conception du temps et de l'être spécifique à sa condition: "Je me sentais autant, sinon plus lié aux générations du passé et du futur qu'à mes contemporains. C'est ma mort que j'appréhendais. Je me replaçais, je me situais dans le temps d'une autre façon." (217) Manifestement, le je scripteur échappe au conditionnement rigoureux et à la nécessité du récit factuel au moyen de l'imagination et de l'écriture: "Vrais ou faux, authentiques ou non, les mots avaient le pouvoir de me faire dévier de ma trajectoire, et ils ont réorienté l'usage du temps qu'il me restait à vivre." (57) Opposée à la profession monotone du dentiste, "[ancrée] dans le tracé droit et simple de la courte histoire" (124), la vie de l'écriture est décrite comme une déviation par rapport à la trajectoire rectiligne d'une existence allant de soi. Le syntagme "démarche du crabe" dont le sens littéral renvoie à un mouvement latéral et oblique se fait la métaphore de l'itinéraire sinueux du protagoniste qui voit resurgir çà et là des souvenirs de 
jeunesse. À plusieurs reprises, le narrateur insiste sur le fait qu'il abhorre sa profession, perçue comme factice et modelée sur du vide: "Je n'avais pas jusque là envisagé la réalité en face: je ne voulais plus faire ça. Réparer des dents. Je ne voulais plus être dentiste. Je n'aimais pas ça. Je n’avais jamais aimé ça" (18); "Je n'avais jamais voulu être dentiste. Je détestais les dents" (43); "réparer des dents, non. Je ne pouvais plus faire ça" (38), autant d'énoncés qui demeurent significatifs de la dimension construite du statut professionnel d'un homme convaincu de "s'être trompé de vie". Par le truchement d'un discours répétitif, le narrateur s'objecte à une existence qui lui semble étrangère et insignifiante : "Je m'étais trompé de vie" $(37,47)$; "Si je m'étais trompé en me mariant, en devenant dentiste, en m'installant à TMR, j'étais censé être qui?» (100)

Loin de se réduire à une vocation manquée, la vie du dentiste montréalais bascule complètement au printemps de l'année 1987. Son existence dans une banlieue chic, auprès de sa femme Nicole, ne revêt plus aucun sens à ses yeux. Désorienté, Santerre est plongé au creux de l'incertitude ontologique dans la mesure où il ne conçoit son être que dans l'indétermination: "Ce qui avait été construit se déconstruisait" (157), prétend le narrateur alors qu'il songe à son mariage. Suite à l'irruption inattendue d'une jeune patiente qui le reporte sans cesse dans ses souvenirs, le je narrant ne se reconnaît plus. Il formule ainsi la déconstruction de son moi : "cette identité que nous tissons fil par fil, choix par choix, sans y être forcé, censée nous aller comme un gant, cette identité s'est mise à se défaire morceau par morceau. Elle tombait comme un costume quand la pièce est finie." (37) Or, la jeune patiente se nomme Sarah Rock. Selon le narrateur, la rencontre de Sarah demeure décisive. Inaugural de l'action romanesque, l'événement fait en sorte "[qu'un] circuit oublié [va] se rétablir" (14) dans la mémoire du protagoniste. Il aura suffi d'un seul regard pour que le personnage masculin comprenne que l'identité n'intervient pas dans une logique fixiste: "[Sarah] m'arrachant mon identité comme on décollerait d'un coup une pellicule de plastique invisible." (14) Autrement dit, la turbulente Sarah incite le professionnel à faire tomber ses masques. Nombreux en effet sont les énoncés qui évoquent l'aspect mouvant d'un être qui s'autoreprésente en usant de métaphores tirées d'un langage se référant aux choses matérielles: "tout le moule avait commencé à craquer" (14); "ma vie était en train de se détacher 
de moi, comme un ongle qui se décolle et vous fait souffrir" (40); "tout cela m'avait quitté, tombant comme les écailles d'un animal qui mue" (51). À partir de ces lieux discursifs, le protagoniste se représente comme une partie intégrante du monde naturel toujours exposé aux changements. Si le narrateur suggère ici un trouble de la mémoire, c'est pour montrer la discontinuité et l'inconsistance identitaires, à l'image de la disparité des phénomènes naturels. Suivant la négation d'une logique binaire où rivalisent le même et l'autre, le personnage s'adonne à l'écriture affirmée comme un lieu de l'expérience façonnant l'existence à divers degrés de réalité. C'est donc à la veille de mourir que le romancier improvisé entreprend son périple au cour de la fiction du temps dont il relate les principaux événements au sein de ses manuscrits.

Si naturelle semble-t-elle définie à l'intérieur du texte de Santerre, l'activité d'écriture demeure intimement liée à la rencontre de Sarah Rock quelques mois plus tôt au cabinet du dentiste: "Je ne savais pas encore que Sarah venait d'entrer dans ma vie pour n'en sortir que par la voie royale du livre que j'écris pour elle [sic]." (21) À son contact, Santerre avoue "[être] insensiblement déporté, ou reporté, vers l'écrit. Vers le murmure diffus de l'écrit [...]. Vers l'implosion d'énergie de ces petits signes" (31). Au terme de ses visites, la jeune fille finit par divulguer ce qui, en fait, la lie au passé du dentiste: Sarah confie à ce dernier qu'elle est la fille de Michelle Roche dont elle a anglicisé le nom. Or, Michelle est une amie d'enfance de Luc-Azade Santerre. Tout se passe comme si le je narrant pénétrait graduellement les couches épaisses du passé dont le sens demeure en partie insaisissable: "Dans la lettre de la fille, j'entendais l'écho de la mère. L'énigme venait de reculer d'un cran. Un noud était atteint: le rhizome principal, une souche plus profonde." (63) La référence à l'arbre généalogique de Sarah s'articule dans le texte du romancier au moyen de l'image du rhizome. Conformément à la logique deleuzienne, ce concept nie l'existence d'un sens univoque. Plus précisément, le rhizome rend compte du réel comme événement et expérience. Il ne l'apparente point à un ordre stable d'objets immuables. Contrairement à l'arbre de la raison cartésien, les pensées-rhizomes ne reconnaissent aucun surcodage unificateur. Envisagées sous un angle pragmatique, elles permettent de "relativiser la référence globalisante et réintroduire une dimension de subjectivité au sein des lois de la logique clas- 
sique postulant que la référence est la même pour tous les sujets qui sont, eux aussi, identiques sous l'angle d'une raison une et universelle" ${ }^{14}$. Singulier et équivoque, l'arbre généalogique commence à se ramifier puisque Rose, la mère de Michelle, s'avère la demi-sœur de Blanche, la mère de Santerre. C'est l'information généalogique dont prend connaissance le Montréalais mal en point, en visitant les lieux du passé remplis des signes par lesquels s'entrechoquent les souvenirs.

Comme l'allègue la pratique autoréférentielle, la chaîne humaine où se situe le protagoniste se présente comme une filiation féminine: à partir de l'histoire de ses prédécesseurs, à savoir la mère et la grand-mère, le personnage masculin est en mesure de relire des pages de l'enfance et de recréer celle de sa mère. Mais la filiation ne correspond guère à une ligne continue, à un "tracé rectiligne". Au contraire, les avatars des générations galvanisent l'enquête de Santerre qui prend en charge plusieurs données aléatoires afin de débrouiller le mystère de ses origines. C'est dans le chapitre "Rive nord" que le dentiste apprend qu'il partage des liens de parenté avec Michelle Roche. Lors de sa visite chez un notaire de La Malbaie, Luc découvre le passé de sa grand-mère maternelle. Selon des renseignements communiqués par la mère du notaire, l'aïeule avait connu une fin prématurée. Emportée par une méningite, quelques jours après avoir donné naissance à une petite fille, la femme était célibataire: "Ma grandmère ne savait ni lire ni écrire mais elle était très belle" (189), note un Santerre placé devant le flou des événements. Il se rend tout de même à l'évidence que "dans un puits sans fond se trouvait la définition de ce [qu'il] était" (191). Sous la forme du discours indirect, le texte indique que la famille Gagnon, les parents de Rose plus exactement, avait recueilli le bébé en couches. Toujours suivant les explications de la mère du notaire, on ne leur avait guère laissé le choix puisque le père de l'enfant était, à ce qu'on a prétendu, Arsène Gagnon. Ainsi que l'atteste le je scripteur au sein des carnets renvoyant au périple sur la rive sud, la région située sur la berge opposée ne comportait aucun attrait pour la mère de Santerre, soucieuse de rompre avec le lieu de ses origines et d'oublier le roman de sa naissance: "Je ne sais rien de ma mère, je n'ai jamais cherché à savoir quoi que ce soit d'elle" (89), avait-elle avoué plus jeune à son fils. En revanche, le

14. Gilbert Hottois, op. cit., p. 419. 
récit autoréférentiel se donne pour tâche de déterrer les morts du fond des âges. L'instance narrative précise que sous les apparences d'une "vie humble et anonyme [...] bâtie artificiellement de toutes pièces sur le vide" (48), Blanche a transmis à son unique descendant "un héritage [...] par-delà sa volonté" (93). Bien qu'une rupture se soit opérée dans l'axe des générations, la transmission du passé a eu lieu. Orpheline, la fillette a grandi parmi la famille de la mère de Michelle. Très jeune, elle a quitté la campagne pour aller s'établir à Montréal où elle a épousé un pâtissier affecté à un hôpital. Ce dernier allait devenir le père de Luc. Selon le je narrant, l'amitié de Blanche et de Rose s'est prolongée jusqu'à ce que les origines paternelles de l'orpheline soient divulguées en 1970. Antérieurement à cette date, Luc et sa mère se rendaient durant les vacances d'été chez Rose, la grandmère de Sarah, comme le raconte Santerre dans "La démarche du crabe".

En dépit de la négation de ses ascendances que lui dicte une croyance en l'anonymat urbain, la mère de Luc "[a] gardé une parcelle du passé dans le présent, une bactérie: son amie Rose, son amour pour le fleuve." (192) De toutes les visites reconstituées quelque trente ans plus tard à l'intérieur des feuillets autobiographiques, certaines images semblent avoir captivé l'attention du jeune garçon dont l'essentiel s'exprime à nouveau par le biais d'énoncés récurrents: "Là-bas ma mère n'était plus tout à fait la femme de mon père. Elle n'était plus tout à fait ma mère" (91); "Elle changeait. [...] Elle surveillait son langage. Elle ne riait plus de la même façon, s'adressait à moi distraitement" (93-94) ; "Là-bas, ma mère n'était plus exactement ma mère" (95); "je crois qu'elle oubliait Montréal et son mari pour redevenir l'amie intime, la "soeur" de Rose" (91). C'est la dimension mouvante de l'être qui domine les extraits précédents puisque la référence dont procède le personnage féminin ne correspond pas à un point fixe et stable. Déracinée une première fois, Blanche est par la suite transplantée dans le décor d'une campagne contrastant avec la ville où elle réside, en compagnie de son mari et son fils. En vertu des réminiscences, le je narrant met en évidence l'emplacement des lieux où se réunissaient les deux amies à chaque saison estivale: "Le fleuve, la maison de Rose, pompeusement baptisée L'Ermitage: des lieux, qui pour ma mère, avaient prestige, grandeur, beauté." (87) En effet, des dissemblances subsistent entre les conditions matérielles structurant la vie des 
deux femmes, si l'on en juge par les propos du père de l'auteur stipulant que Rose appartenait à une classe sociale supérieure à la sienne: "[Rose] était de la "haute classe"." (91) Pareilles différences ont institué une hiérarchie entre les familles dont la génération subséquente reproduit les principaux rouages. Pourvue d'“une vague supériorité des manières" (23), les descendantes de Rose ont "une façon de glisser sur la vie comme des cygnes hautains" (67). Ce qui explique pourquoi Michelle, depuis leur jeunesse, a toujours considéré Luc comme un subalterne: "Ainsi cette amitié de ma mère et de Rose avait-elle engendré de toutes pièces le sentiment non identifié qui me liait depuis toujours à la petite fille tyrannique à laquelle on me livrait chaque été, en juillet." (92) Ce passage est capital: c'est l'interprétation des liens familiaux développés entre Rose et Blanche qui permet au narrateur de faire la lumière sur son passé et de comprendre la nature de son attachement pour Michelle, la mère de Sarah. Comme l'énonce le chapitre intitulé "Rive sud", Santerre visite ces lieux baignés par le fleuve qui deviennent "le plus ancien ici de [sa] vie" (156), d'autant plus qu'ils s'inscrivent dans l'espace archéologique de la jeune fille. La formule "si p alors q" s'applique bien à la proposition modale suivante: si Blanche n'avait pas été liée à ce point à Rose, alors Luc aurait vécu sans connaître Michelle.

L'homme entend réanimer le passé afin de répondre aux attentes de Sarah qui est à la recherche de son père. La présence de la jeune adulte le projette vers les couches rhizomatiques du passé et initie le projet scriptural du narrateur dont les certitudes vont s'émousser progressivement. À l'encontre de la logique aristotélicienne, Sarah existe sans que son père ne fut puisqu'elle ne le connaît point. Quant au je narrant, il se propose de révéler à la jeune fille le secret de ses origines dans une lettre qu'il lui destine. Il acquiesce à la demande de sa nouvelle amie car "[il] estime que tout le monde a droit d'être informé de son passé" (21). Sarah est donc interpellée par le discours consacré aux événements marquants de l'été 1967, relatés au sein de la plus longue section de l'épitaphe. Transposé sous la forme épistolaire, le récit prescrit par définition la présence d'un $t u$ : "Je vais raconter exactement l'été qui précède ta naissance." (111) Mais la narrataire est désormais inaccessible. Ce que produit la narration des événements, c'est une lettre dont l'accumulation de signes matérialise la préhistoire de Sarah. À défaut de parvenir à la destinataire, la missive s'accorde au projet discursif pris en charge 
par le moi prolixe de Santerre: "me parler à moi-même en te parlant." (147) Sur le plan de l'énonciation, le texte dérive en effet de son sens initial dans la mesure où le je épistolier transgresse les registres de la représentation: "Je" n'existais plus. Je n'avais plus à me supporter moi-même." (103) Le schéma de communication proposé occulte un besoin autre que celui de divulguer l'identité du père à Sarah. Suivant la pratique autoréférentielle de l'écrit, il importe pour le narrateur de se remémorer les événements qui ont délimité une frontière temporelle entre ce qu'il considère comme l'avant et l'après. La période où s'est tenue l'Exposition universelle érige en fait une ligne médiane entre la "préhistoire" et l'"histoire" de Santerre. L'épisode charnière concerne les circonstances entourant la rupture de Luc et de Michelle en octobre 1967. À la période qui correspond aux premières années vécues près de Michelle - la préhistoire - en succède une seconde - l'histoire - dont le début coïncide avec le mariage de l'universitaire et de Nicole, la mère de ses enfants. Ce qui est déconstruit, c'est "l'histoire", conditionnée par l'hégémonie de l'ordre social établi. Après avoir fait la connaissance de Sarah, le personnage gomme ce qu'il a été durant les vingt dernières années de sa vie. On comprend mieux dès lors que le je scripteur s'applique à fouiller les strates préhistoriques du passé dans le but de reprendre contact avec ses gênes archaïques. En exergue à "L'Exposition" se trouve un extrait du roman Les vagues. Rappelons que le texte de Virginia Woolf, publié en 1931, est fondé sur le thème du temps: "La planche du présent était étroite, et le torrent dessous faisait rage." (105) Or, de cette configuration spatio-temporelle surgit un réseau de significations engageant le récit rétrospectif dans les remous du passé qui, sous bien des angles, n'est guère solidaire du présent. Vraisemblablement, le brouillage autoréférentiel, instauré par la méditation du personnage au sujet de la première moitié de son existence, débouche sur une dérive du sens au sein d'un message épistolaire dont le statut du destinataire n'est pas parfaitement établi.

En dépit des structures floues de la mémoire, Santerre identifie la présence d'un signe clair, susceptible d'aider la fille de Michelle à retrouver son père. Ce dernier se nomme Marc Martin. Il s'agit d'un homme de souche amérindienne depuis le côté maternel. À l'été de l'année 1967, il était devenu l'amant de Michelle Roche. Luc, qui a partagé l'intimité du couple, a donc 
fréquenté assidûment Martin. Au fil de leurs discussions, ce dernier a fait part à son ami de ses idées sur le monde en général et plus particulièrement sur l'Amérique décrite comme "la terre du métissage" (124) par excellence. Percevant le monde comme "un vaste mélange ethnique" (124), Martin a toujours adopté une position des plus fermes en ce qui a trait à l'unité et la continuité de la race. Issu de la plurielle "terre des hommes", le métisse ne voulait pas devenir père. Tributaire des renseignements que sa mère lui a confiés, Sarah rapporte ce qu'elle sait de son géniteur dans une lettre expédiée à Santerre: "cet homme ne voulait pas de descendants. Quand il avait appris qu'il allait être père, il avait fait jurer à Michelle, sous peine de mort, de ne jamais dévoiler son nom à cet enfant" (60). Sous l'effet de telles menaces, Michelle a préservé l'anonymat du père de son enfant. Mais Martin a transmis à Sarah une maladie due à une mutation héréditaire: le père et la fille ont des saignements de nez subits, un gène particulier qui apparât, aux dires du narrateur, tel "un signe de filiation". Comme l'écrit Santerre, il s'agit d'"un marqueur génétique" (147) car "les gens atteints ont tous un ancêtre commun" (147) ${ }^{15}$. Pareils signes connotent le même et signalent une continuité à travers les générations. En dépit de l'aspiration de Marc Martin à confondre son être singulier dans la multiplicité humaine, un phénomène d'ordre physique montrant une pathologie définie le lie pour toujours à Sarah Rock.

Parallèlement aux prétentions à l'universalité du métisse, les critères sur lesquels se fonde Santerre pour décrire sa progéniture tiennent plutôt de liens dénaturés. Il est le père de deux enfants auxquels il prétend n'avoir rien transmis: "Je ne me retrouvais pas en eux." (43) Plus encore, l'auteur perçoit son garçon et sa fille comme des "spécimens parfaits de l'humanité de race blanche" (43), des êtres "sans peur et sans angoisse" (43), en symbiose avec la socioculture dont ils proviennent. Sur un ton tranchant, il les qualifie de "barbares" puisqu'ils sont détachés de toute connaissance historique. Comme l'évoque le récit spéculaire, la structure de parenté abonde dans le sens de la rupture et de la discontinuité. Si l'on en juge par les occurrences du mot "générations", la question généalogique comme vecteur de l'identité obsède le narrateur. Les expressions suivantes en sont la

15. L'auteure fait ici référence à un trouble de coagulation d'ordre héréditaire qui s'apparente à la maladie de Von Willebrand. 
preuve éloquente: "mondes soumis à la génération et à la corruption" (80), "séparer les générations" (102), "le contact des générations" (123), "après plusieurs générations en Amérique" (153), "le mouvement des générations" (158), "imaginer le plaisir des autres générations" (193), "les générations se reliaient, se répercutaient " (210), "après des générations en nombre suffisant" (212). Par voie de conséquence, la corruption de la génération culmine dans le texte en introduisant l'idée d'un espace-temps qui brise le mouvement cyclique et naturel de la génération: "On ne peut pas être et avoir été" (96), se souvient Santerre, fidèle lecteur d'Aristote dans sa jeunesse. Aussi chaque étape du voyage est-elle reproduite suivant le modèle d'un parcours narratif se dédoublant et rompant avec un simple mouvement linéaire, une continuité et une logique de non-contradiction: "J'étais un crabe. En marche vers un trou plus ancien. En déséquilibre entre deux carapaces. [...] Un être hybride ni chair ni poisson." (72-73) La démarche en zigzag symbolise le dédoublement à l'intérieur de la mémoire dont les structures répétitives ponctuent fréquemment l'énonciation. Tout se passe comme si la mécanique textuelle oscillait entre les pôles du même et de l'autre, compte tenu de l'hybridation d'un sujet comparable à un crabe et du déséquilibre caractérisant sa démarche ${ }^{16}$. Le je scripteur va métaphoriser et illustrer le jeu de cette altération spatiale dans son roman à l'aide de philosophies ayant trait à la structure inchoative de l'identité.

16. Mentionnons que l'image est présente dans Les fous de Bassan d'Anne Hébert. Stevens Brown, l'une des instances narratives, décrit la promenade sur la grève de son oncle, le révérend Jones, comme une errance similaire à celle de Santerre en quête de son passé: "[...] sa démarche sur le sable est celle d'un crabe qui ne sait où aller " (Anne Hébert, Les fous de Bassan, Paris, Seuil, 1982, p. 106). La procédure intertextuelle est d'autant plus percutante si l'on examine un fragment du même ouvrage, mis en exergue au premier chapitre du roman de LaRue: "Laissons les souvenirs disparaître dans le sable à la vitesse des crabes creusant leur trou "(La démarche du crabe, p. 7). Il s'agit cette fois d'un passage puisé à même le récit sans date de Olivia de la haute mer (Anne Hébert, op. cit., p. 206-207). Telle qu'articulée dans le texte hébertien, la métaphore d'un passé inaccessible est prise en charge par une voix narrative se référant à un personnage décédé. On saisit les liens qui se tissent entre les ouvres romanesques respectives évoquant l'altération physique de l'être plongé dans l'abîme du temps. 


\section{L'atomisme logique : la relativisation de l'Un et du Multiple dans l'écriture}

Comme l'a montré la première section consacrée à l'autoreprésentation textuelle de l'identité, c'est une altération physique de l'être qui module l'écriture du passé dans La démarche du crabe. Les structures autoréférentielles de l'énonciation conduisent à une lecture de l'énoncé confondant sans cesse deux conceptions de l'être. En l'occurrence, la traduction des savoirs permet de cerner l'articulation de ces paradigmes à l'intérieur d'un récit dont l'objectif consiste à décrire la création littéraire du personnage principal en fonction d'une conception esthétique du temps et de l'espace. Il s'agit de la quête d'un sujet scripteur engagé dans le processus "de la génération et de la corruption" de l'identité alors que, condamné à mourir, il entreprend de signer son premier et dernier livre. L'énonciation décrit le mouvement des générations comme un phénomène complexe, discontinu, hasardeux puis le met en rapport avec un principe d'identité qui, par définition, rend compte de la permanence de l'être. Il importe ici d'identifier les thèses philosophiques mises à contribution dans "le roman à venir" pour traiter du principe dynamique d'individuation. Ces thèses concernent principalement la permanence dans le changement. Les effets signifiants de la transtextualité romanesque permettent d'appréhender l'identité comme quelque chose de calqué sur la nature qui, elle-même, se compose d'une matière non immuable.

Sur le plan de l'énoncé, le processus d'individuation s'articule autour d'un intertexte bien circonscrit. Il s'agit de savoirs qui émanent d'abord de doctrines philosophiques issues de l'époque présocratique. Orientées vers une réflexion portant sur des phénomènes physiques (phusis) ${ }^{17}$, ces écrits philosophiques tentent de penser l'ordre et le désordre de la nature, posée comme une réalité à la fois une et multiple. Les principes matérialistes auxquels adhère l'atomisme antique s'inscrivent dans la tradition de pensée de Démocrite ${ }^{18}$ dont les disciples les plus influents furent

17. "Phusis est le premier nom philosophique de l'être [...]. Phusis est la matière universelle, vivante, qui croît, se développe et s'épanouit partout diversement, en tout ce qui est et qui devient". De l'époque présocratique qui marque la naissance de la philosophie grecque sont issus "des bribes de traités dont la plupart étaient intitulés: Peri Phuseôs ("Au sujet de la nature")". Gilbert Hottois, op.cit., p.14.

18. Démocrite serait né vers 460 av. J.-C dans la ville d'Abdère. Il serait l'auteur d'une œuvre immense et encyclopédique dont il ne reste que 300 fragments. 
Épicure et Lucrèce ${ }^{19}$. Ce retour à une tradition matérialiste, dont l'ébauche fut esquissée il y plus de deux mille ans par les philosophes de la nature, ne comporte rien d'étonnant dans le manuscrit d'un homme qui avoue faire de la philosophie comme on fait de la cuisine. Passionné pour la spéculation et la poésie, le dentiste a suivi des cours à la télé universitaire qui lui ont révélé entre autres les textes d'Héraclite, de Parménide et de Démocrite ${ }^{20}$. Aussi Santerre présente-t-il, dès le début du chapitre intitulé "La démarche du crabe", le discours des philosophes présocratiques débouchant sur la question de l'identité unitaire, mise en rapport avec la diversité des phénomènes éphémères. Dans un premier temps, le narrateur se réfère à l'allégorie d'Héraclite: "On ne se baigne jamais deux fois dans le même fleuve." (79) Au sein du livre Sur la nature, le philosophe ébauche une réflexion portant sur le devenir. Il voit dans le mouvement ce qui demeure identique à travers la diversité des êtres. Héraclite postule que l'identité est toujours mouvante, oblique et sans fondement. La connaissance de soi s'avère impossible dans la mesure où il n'y a pas de continuité dans notre existence. C'est ce que constate le je narrant lorsqu'il affirme: "Je n'ai plus rien à voir avec celui que j'ai été." (79) Selon Héraclite, il s'agit du Multiple, de l'impossible identité soumise irréductiblement aux lois du changement. À l'opposé, les textes de Parménide font état de la fixité de l'identité s'érigeant en vertu d'une logique fondée sur la permanence de l'intelligible. De la conception de l'être élaborée dans De la nature découle le

Dans Le petit système du monde, le philosophe grec expose la doctrine de l'atomisme. Avec son compagnon Leucippe, il développe les rudiments de la physique.

19. Né vers 341 av. J.-C, Épicure situe sa philosophie dans le prolongement de la doctrine de l'atomisme. Au sein de la célèbre Lettre à Hérodote sur la physique, il expose les fondements de ses thèses matérialistes. Philosophe latin du I $^{\text {er }}$ siècle avant notre ère, Lucrèce a transposé l'essentiel du matérialisme d'Épicure dans un poème exposé en six livres, De la nature des choses.

20. Ayant vécu aux alentours de 530 av. J.-C., Héraclite serait l'auteur d'un livre dont ne subsistent que des fragments. Le titre en est Sur la nature. Il s'agit d'un tableau explicatif des différents phénomènes que l'homme peut observer dans l'Univers. Quant à Parménide, il aurait écrit un poème en hexamètres dont 55 vers seulement nous sont parvenus sous le titre De la nature. Il aurait vécu à la fin du $\mathrm{VI}^{\mathrm{e}}$ siècle avant notre ère. Héraclite, Parménide et Démocrite figurent parmi les premiers représentants de la pensée grecque. Voir Abel Jeannière, Les présocratiques. L'aurore de la pensée grecque, Paris, Seuil, 1996. 
principe d'identité formalisé par A est A. Inflexible, cette structure rejette le couple du même et de l'autre. À la faveur de la doctrine des Éléates, la connaissance est rendue possible par la continuité. L'adage "tel père, tel fils" illustre bien la constance dont il est question ici. Partisan d'une ontologie statique, Parménide stipule avec l'unité de l'être, l'Un, c'est-à-dire un concept d'identité qui se fonde sur des certitudes et la connaissance. Démocrite vient enfin résoudre le paradoxe de l'Un et du Multiple énoncé par les penseurs précédents en prétendant que "l'univers est constitué d'atomes insécables et permanents". Suivant l'interprétation de Santerre, la thèse de Démocrite se résumerait à ceci : "On change mais on reste soi-même. On est seul, enfermé en soi-même, et pourtant les autres pénètrent continuellement la membrane qui nous constitue et nous sépare d'eux." (80)

À la lumière des leçons philosophiques fièrement mémorisées, Luc-Azade Santerre décrit ses rapports avec Sarah et Michelle Roche, rapports qui demeurent déterminants au sein de sa "démarche du crabe". Inspiré de la thèse d'Héraclite selon laquelle le changement est un principe d'identité, le narrateur conclut que l'homme qui avait tant aimé Michelle plusieurs années auparavant n'existe plus. Santerre évoque ainsi la transformation incessante des choses et des êtres. Par ailleurs, la pensée éléatique de Parménide rend possible la présence d'un moi permanent, produit par la juxtaposition du passé et du présent. Ce philosophe reconnaît la nécessité d'une identité absolument pure. À sa manière, Santerre traduit les propos de l'ardent défenseur de l'identité logique: "Sarah et Michelle avaient réveillé [le moi, le substrat]" (80). Cependant, c'est sur la thèse atomistique, déterministe et matérialiste développée par Démocrite que s'appuie Santerre pour établir le fil conducteur de son récit. Selon le penseur présocratique, les atomes sont des entités numériques tenues pour des réalités individuelles et singulières. Le je narrant souscrit à l'idée que l'être est matière: un atome indécomposable et doté de mémoire, lui-même déterminé par l'espace-temps. Plus précisément, la doctrine des atomistes stipule que la matière est formée d'atomes. Comme le mentionne Santerre, l'atome est indécomposable, à savoir incorruptible et incréé. C'est l'Un renvoyant à la permanence de la matière. Élément primitif et fondamental, l'atome est donc l'incarnation physique minimale de la matière. Or, la création des corps matériels résulte de combinaisons fortuites dans le vide infini. L'auteur du Petit système du monde 
132

soutient que la trajectoire perpétuelle et tourbillonnaire des corps élémentaires dans le vide produit des collisions entre les atomes. C'est le Multiple assorti à la loi principale de la matière: le mouvement. La dimension déterministe de l'atomisme dont s'inspire l'auteur Santerre est donnée dans le postulat suivant: l'éternel mouvement des atomes est cause de tout. Comme l'indique le narrateur, la Fortune fait en sorte que les atomes forment un monde parce qu'ils tombent ensemble à un moment et dans un lieu précis: "Et moi j'avais été parachuté au même instant et au même endroit que Michelle Roche." (81)

La philosophie élaborée par Démocrite émerge du texte qui se construit suivant une conception atomistique du monde: "Tout ce qui existe dans l'univers est le fruit du Hasard et de la Nécessité", affirme le citoyen d'Abdère. La nécessité — anànké — est la contrainte universelle qui pousse sans cesse la matière à se mouvoir tandis que le hasard - tukné — est la contingence. Comme le rappelle Jean Largeault, "les Grecs croyaient à la nécessité et en distinguaient deux sortes, l'une que la raison saisit, l'autre qui lui échappe: la nécessité aveugle, i.e. opaque à l'intelligence et que ne dirige aucun dessein" 21 . C'est donc la transposition romanesque des entrecroisements discursifs possibles du hasard et de la nécessité qui constitue le fil narratif de Santerre. Telle qu'elle s'imbrique dans le roman spéculaire, la relation du personnage narrateur avec Michelle Roche est révélatrice d'une conception du monde héritée des premières philosophies de l'être où l'on tente d'expliquer la nature à partir des seuls principes physiques. Si l'on s'en remet à l'intertexte déployé au sein du manuscrit de Santerre, il y aurait une double existence objective du hasard et de la nécessité, ce qui souscrirait à la thèse atomistique. Le mouvement de l'atome, faut-il le préciser, est à la fois nécessaire et contingent: contingent dans son mouvement et son lieu, mais nécessaire en tant que propriété de l'atome. À l'image des combinaisons fortuites d'atomes, Michelle et Luc se sont rencontrés. Examinons une première perspective adoptée par le narrateur. Selon ses dires, le hasard explique en partie le fait que les deux individus ont été mis en présence pendant plusieurs années: "Michelle était le signe d’un caprice absolu, de

21. Jean Largeault, "Causes, causalité, déterminisme", dans La querelle du déterminisme. Philosophie de la science d'aujourd'bui, Paris, Gallimard, 1990, p. 177. 
la contingence de la vie. [...] Nos routes s'étaient croisées. Nous provenions du même fonds." (186) Fondée sur la préséance du contingent sur le nécessaire, la thèse du personnage s'énonce ainsi: les êtres au devenir périssable auraient pu exister l'un sans l'autre. Selon cette optique, aucune finalité, aucune force surnaturelle n'intervient en vue de produire le monde formé par les corps matériels. Michelle Roche et Luc-Azade Santerre sont nés le même jour et leurs mères respectives sont des amies intimes. Voici comment le je narrant transpose le phénomène: "Je ne crois pas au destin. Pourtant, ma mère et celle de Michelle se sont mariées le même mois. Elles ont accouché le même jour [...]. À cause de ce hasard, Michelle a tout de suite été considérée comme ma "jumelle". [...] Dans les limbes de l'utérus j'ai été arbitrairement accouplé à cette petite fille." (64-65) Tout se passe comme si une nécessité d'ordre naturel dissimulait le passé. Incidemment, le narrateur parle d'" un sentiment qui avait son origine en amont de [sa] vie" (92). Comme le réitère la voix narrative, il s'agit plus précisément de "liens alambiqués, des liens vivants qui vieillissaient avec [le personnage], et l'éradication ne serait jamais définitive" (66). La dimension arbitraire et accidentelle dont rend compte le procédé de l'autoreprésentation est ici relativisée par un discours concentré sur la fatalité des événements qui serait à l'origine des liens établis avec la "jumelle". Au fil de sa démarche, Santerre attribue à "l'erreur fatale de [sa] grand-mère inconnue" (93) les raisons de son amitié pour la fille de Rose. D'ailleurs, le vocabulaire auquel a recours le sujet scripteur afin de traduire les frasques de Michelle Roche, toujours prédisposée à "faire l'actrice, la femme fatale" (128), implique l'idée de fatum que stigmatise le personnage féminin. Se remémorant une manifestation féministe à laquelle avait participé Michelle au début des années soixante-dix, le narrateur revoit la jeune femme "avançant poitrine nue dans la rue comme une figure de proue, une statue guerrière" (68). Apparentant la croisade de Michelle à une "dérisoire Victoire de Samothrace" (69), il la perçoit avec les attributs de "la tragédienne, la grande actrice" (69). Parce qu'il adopte le point de vue déterministe et causaliste, l'ancien dentiste soutient que "[des] événements qui s'étaient passés avant [sa] naissance [...] avaient influencé [son] caractère." (192) Dans la foulée d'un déterminisme appliqué aux phénomènes naturels, le je scripteur stipule qu'“[il] n'y a pas d'effet sans cause" (115), étant donné que rien n'est le fait du hasard dans la nature où tout est le fruit d'infimes transformations. 
134

Projeté au sein d'un système de référence circonscrit par l'espacetemps, le jeune Santerre que se remémore l'auteur personnifie un être susceptible d'établir des liens causals entre deux états de faits: "Le temps n'avait pas coulé, le temps ne pouvait pas passer. Nous étions encore les enfants du même âge de deux femmes liées depuis l'enfance qui avaient peut-être déjà, je ne sais pas, le même genre de relations autoritaires et infantiles." (115) Autrement dit, les atomes dotés de mémoire reproduisent un monde soumis aux lois objectives d'une nature en devenir. Comme en fait foi l'entreprise romanesque de Santerre, la piste floue et discontinue qui conduit au passé se modèle sur la nature, "au hasard [ou ] à la fatalité [...]. Comme du vent" (46).

Suivant les thèses matérialistes avancées par les Anciens, la seule forme réelle de nécessité est inhérente à la nature et ne relève pas d'un pouvoir divin. Lucrèce et Épicure tentent d'expliquer les manifestations de la nature, tels le tonnerre et les ténèbres, à partir d'hypothèses rationnelles. En ce sens, la combinaison des atomes ne s'opère pas au hasard; ils s'associent en obéissant à des lois. À l'exemple des lois qui régissent la nature, l'homme porte la cause de ses actions en lui-même. Le monde matériel suffit donc à rendre compte de l'homme tout entier, solidaire de la nature. Dans le prolongement de la pensée de Démocrite, Épicure soutient que les atomes ne sont assujettis à rien qui ne soit extérieur à eux. Toutefois, le philosophe rompt en quelque sorte avec le déterminisme inhérent au mouvement rectiligne des atomes exposé par Démocrite. Il évoque l'idée d'une chute diagonale des corps constitués. Il s'agit du clinamen qui rend compte du mouvement oblique des atomes. À l'instar d'Épicure, Lucrèce réfute toute nécessité en dehors du mouvement du corps fondamental. Santerre fait sienne la conception de Lucrèce selon laquelle "rien ne se perd et rien ne se crée" (117), lorsqu'il fait allusion au motif de sa création littéraire: "Tu es le résultat de ce que je te raconte, Sarah, je n'invente rien." (117) Le romancier souscrit ainsi à l'une des thèses fondatrices du matérialisme antique, la permanence de la matière: "rien ne naît du non-être, rien ne retourne au non-être [...]. Tout ce qui périt ne périt qu'en apparence." 22 En accord avec le clinamen d'Épicure,

22. Lucrèce entend démontrer que l'Univers est mortel comme les parties qui le composent. Voir Lucrèce. De la nature des choses. Deuxième édition revue et augmentée. Textes choisis. Préface, commentaires et notes explicatives par Georges Cogniot, Paris, Éditions sociales, 1974, p. 37. 
le poète latin affirme que la déclinaison est la loi de l'atome. En vertu d'une déviation de la ligne droite, le mouvement de la déclinaison est synonyme de hasard, de contingence. C'est à la faveur de la déclinaison des atomes et de leurs rencontres que les premiers corps se seraient formés. On aura tôt fait de constater que le je scripteur multiplie les signifiants du langage qui renvoient au clinamen. À commencer par la forme de la lettre z composant le deuxième prénom du personnage, "Azade". Le z évoque ici la trajectoire oblique de l'éclair qui est un signe de la contingence des éléments naturels. Puis, il y a les mots de l'écriture qui font "dévier la trajectoire" (57). "La démarche du crabe" qui renvoie à un mouvement latéral constitue sans contredit une image retentissante. C'est la métaphore clé dont se sert le narrateur pour décrire son trajet spiralé vers le passé: "J'étais un crabe. En marche vers un trou plus ancien." (72) Le portrait que le texte brosse de Rose, Michelle et Sarah reproduit avec brio l'idée de déviation. Les trois femmes sont posées comme des "générations gigognes" (66). Il s'agit de "femmes diagonales" (73), "indéchiffrables" (21), telle une "énigme" (23-24). Le narrateur signale entre autres "leurs manières inextricables" (59), "leur manière oblique, indirecte de procéder avec les autres" (67).

$\mathrm{Vu}$ sous cet angle, le retour à des origines, ne pouvant se fonder sur aucun point organisateur, exprime la relativisation de l'un et du multiple dans les manuscrits de l'ancien dentiste: "Je ne savais pas qu'écrire modifie celui qui écrit. Je ne serais plus exactement le même ensuite. Continuité, changement ne s'opposeraient plus autant quand "l'encre aurait coulé sur le papier" " (103). Comme l'indique Michel Serres dans La naissance de la physique dans le texte de Lucrèce, "les atomes sont des lettres ou sont comme les lettres. Leur entrelacement constitue le tissu des corps, de la même manière que les lettres, entre elles, forment des mots, des vides blancs, des phrases et des textes "23. Suivant un procédé matérialiste, l'atomisation de l'écriture se concrétise progressivement dans le texte romanesque de Santerre. À l'image du clinamen d'Épicure rendant compte de la déclinaison des atomes et de leur bifurcation contingente due au hasard, les traces laissées par l'écrit illustrent la mise en abyme du sujet agonisant qui, désirant se faire autre, tente également de produire un

23. Michel Serres, La naissance de la physique dans le texte de Lucrèce. Fleuves et turbulences, Paris, Éditions de Minuit, 1977, p. 175. 
136

sens différent. Le regard rétrospectif vise alors à dégager ce sens, à repérer un fil d'Ariane à travers le labyrinthe des souvenirs. Inspirée de l'atomisme gréco-latin, la réflexion philosophique et littéraire amorcée par Santerre l'amène à considérer son identité comme une fiction dont le centre ou le noyau atomique est nulle part et la circonférence partout. Posé comme simulacre, le moi se métamorphose au gré des intempéries. À l'inverse, la pratique scripturale devient symbole d'évasion et de survie, tel un navire d'espoir dérivant sur un fleuve d'encre et de signification qui laisse advenir une singularité de principe: "Un livre est un objet relativement difficile à détruire. [...] Peu, très peu de choses sont aussi résistantes. Le résidu, les pépites d'or des fleuves de mots que j'ai lus et admirés depuis ma jeunesse m'ont aidé à surnager" (217). Ce passage évoque la consistance de la culture qui donne une assise à l'identité du protagoniste. La métaphore du fleuve, déjà associée aux lieux de l'enfance, demeure significative de la constitution et de la dérive du je narrant, compte tenu du dédoublement spéculaire engendré par l'écrit. Investi dans la pratique scripturale élaborée à partir des lieux de mémoire, le moi hybride de Santerre reconstitue puis remonte le fil identitaire le liant pour toujours à Sarah et à Michelle Roche.

\section{Théorie du chaos et hasard déterministe : la réduplication de l'identité postmoderne}

Devenu un élément quasi structural du réel, le hasard est considéré comme intrinsèque aux phénomènes physiques. Nous avons déjà montré que les Anciens avaient formulé cette question dans le contexte des philosophies de la nature aux fondements matérialistes. Or, des thèses récentes, avancées par les défenseurs de la théorie du chaos, examinent la perspective d'une géométrie de la nature au moyen de savants calculs statistiques. De concert avec Patrick Brady, mentionnons que "la théorie du chaos est la plus récente des théories scientifiques à être appliquée au domaine littéraire [et qu'elle] a comme source lointaine les recherches topologiques publiées vers la fin du dix-neuvième siècle par le mathématicien Henri Poincaré „24. Dérivée des mathématiques et de la physique, la théorie du chaos réside dans la capacité

24. Patrick Brady, "La théorie du chaos et L'œuvre: peinture, structure, thématique", Les cabiers naturalistes, n 66, 1992, p. 105. 
pour un système dynamique de créer des comportements aléatoires, des turbulences. On classe dans cette théorie une série de phénomènes physiques obéissant à des lois parfaitement déterministes mais dont les systèmes d'équation ne sont pas linéaires. Nourri d'un intérêt accru pour le chaos et le désordre, ce domaine de la science s'écarte résolument des préférences platoniciennes pour les Formes pures. Prenant de multiples ramifications, ce discours scientifique est introduit dans le récit de Santerre qui, comme nous le savons, pose le hasard comme un complément et une manifestation de la nécessité, une forme sous laquelle apparaît le lien objectif des choses. Quatre principes formels, faut-il le rappeler, constituent l'essentiel de la théorie du chaos. Le premier a trait à la dynamique ordre/désordre: il concerne l'ordre dissimulé derrière une apparence de désordre. En second lieu, le hasard contraint renvoie au mouvement erratique à l'intérieur de limites établies. Vient ensuite la non-linéarité qui rend compte de la disproportion entre cause et effet, symbolisée par l'effet papillon. Finalement, on trouve l'auto-ressemblance, typiquement représentée par les fractales. Ces quatre principes sont à l'œuvre dans la narration de Santerre.

Du début à la fin de son texte, le romancier établit de façon explicite un dialogue entre l'identification rationaliste de l'ordre et les formes aléatoires du désordre: "Les mots se gravaient en moi, transformaient le désordre créé par Sarah en un ordre nouveau." (66) Rien d'étonnant alors si une "[t]race d'ordre dans le désordre" (213) spatialise le récit. Engendré par des brisures de symétrie, le désordre devient créateur. Tout se passe comme si l'aléatoire jouait un rôle crucial dans les mécanismes de structuration du réel. Le discours romanesque montre spécifiquement comment s'actualise un ordre unifié qui relève des apparences. De fait, l'épisode où Santerre relate la mort de sa mère accomplit au plan discursif "le dialogue de l'ordre et du désordre" 25 . Derrière le paravent d'une vie humble et anonyme se dissimule un passé bouleversant: "Ma mère m'avait toujours paru s'insérer harmonieusement dans l'ordre des choses. [...] En apparence, la mort était simple." (48) Mais il n'en est rien, comme le corrobore la visite du personnage écrivain à La Malbaie. Déjà fasciné par les

25. L'expression est empruntée à Edgar Morin. Voir "Le dialogue de l'ordre et du désordre", dans La querelle du déterminisme, Paris, Gallimard, 1990, p. 79101. 
138

textes fondateurs de la physique atomistique, l'auteur des manuscrits témoigne d'un intérêt frappant pour la question relative à l'ordre qui "permet de comprendre que les contraintes, invariances, régularités dépendent de conditions singulières ou variables" ${ }^{26}$. Edgar Morin a largement commenté le dilemme auquel sont confrontés aujourd'hui les scientifiques tâchant de "concevoir l'association et la coopération de l'aléatoire et du déterminisme" ${ }^{27}$. Selon l'épistémologue, "ce qui est seul réel, c'est la conjonction de l'ordre et du désordre "28. Or, Santerre situe son propos dans cette perspective dialogique et subjective alors que dans ses carnets "il renonce au déterminisme ontologique [et] s'ouvre à l'idée que notre logique, nécessaire, est insuffisante pour concevoir la richesse du réel " 29 .

À ce premier principe de l'ordre caché et/ou apparent fait écho celui du hasard limité ou contraint qui le prolonge d'une certaine manière. À travers le manuscrit, les notions de contingence et d'accident sont mises en discours sous de multiples formes. Il faut dire que le signifiant "hasard" avec les mots qui forment son champ sémantique — "fortuit " contingent" "accidentel" —, jalonnent le récit. Or, la présence de ce vocabulaire se fonde sur un savoir spécifique. Rappelons qu'il incombe aux théoriciens du chaos de soumettre à l'investigation des phénomènes météorologiques qui traduisent la difficulté de prévoir les événements de façon univoque. Visiblement, les phénomènes naturels connotent l'imprévisible. Au début de son roman, Santerre a recours à des éléments climatiques pour introduire l'idée de la contingence. Il signale en effet l'arrivée pour le moins déconcertante d'une tempête hivernale au mois de mai: "J'aime les orages et les tempêtes, le vent, le grésil et tous les signes météorologiques. Ils nous rappellent que nous ne dirigeons rien et que nous sommes contingents." (12) La dernière partie du récit, qui porte sur les aventures du protagoniste en forêt, renvoie à l'idée d'un chaos déterministe et met en relief la dimension contingente de l'existence humaine. De fait, le narrateur lie l'ensemble de son projet d'écriture à l'imprévisible, au hasard: "Si je n'étais pas allé chez Therroux je n'aurais pas écrit. Tout est contingent. Rien n'est

26. Ibid., p. 83.

27. Ibid., p. 81.

28. Ibid., p. 81.

29. Ibid., p. 91. 
nécessaire." (217) La fiction expose une conception de l'univers qui se fonde sur "les modèles les plus simples du déterminisme et de l'aléatoire: la nécessité rationnelle des Grecs (logos) et la déclinaison des atomes (la cause libre, l'innovation)"30. À l'instar des présocratiques, le sujet scripteur nie un ordre gouverné par une puissance extérieure. À la recherche d'autres signes dans la profondeur des bois, il demeure attentif au discours de celui qui l'accompagne à la chasse, chaque automne. Ancien médecin devenu guide en forêt, Gaston Therroux vit dans une cabane "au désordre minutieux" (204). Retiré sur une île, il dispose de savoirs utiles et pragmatiques. Therroux fait comprendre à son hôte que "[les] redoublements, [les] effets de miroir ne sont pas des hasards, mais des manifestations d'ordre, logiques dans un univers fermé, qu'il soit grand ou petit" (208). Bien que les choses ne se répètent jamais de façon identique et ne découlent d'aucune nécessité, les apparitions successives de choses différentes souscrivent à un ordre dans la mesure où elles surviennent au sein d'un même système de référence. Il devient alors possible de dégager une régularité des fluctuations, l'identité du devenir, l'un du multiple. Aussi la représentation de l'identité passe-t-elle ici par la métaphore d'un mouvement erratique puis évoque à maintes reprises l'idée d'un hasard contraint par des paramètres objectifs.

À l'encontre de la logique classique qui postule un ordre de causalité stricte, la théorie du chaos n'établit pas de lien nécessaire entre la cause et l'effet. Il s'agit du principe de non linéarité qui met en lumière la disproportion entre la cause d'un phénomène et les effets escomptés. Le texte de Santerre foisonne d'expressions qui amplifient ce type de rapport. Pensons aux passages où le je scripteur s'étonne de l'incidence de Sarah sur le cours de son existence réduite à néant: "Ce que je venais d'apprendre m'avait instantanément transformé." (63) Le narrateur distingue alors les lois de la causalité, selon lesquelles on doit "s'en tenir à des décisions simples [et suivre] le fil des causes et des effets" (89), puis d'autres plus complexes par lesquelles on remonte péniblement le cours du temps. C'est le sens conféré par le je scripteur aux procédés mémoriels de l'autoreprésentation où passé et présent communiquent. À en juger par le discours narratif, cerner ce qui définit les êtres, selon une perspective rationnelle et déterministe, demeure une tâche laborieuse: "des valeurs et des

30. Jean Largeault, op. cit., p. 177. 
140

conduites qui venaient de loin avant nous dictaient depuis longtemps ce que nous étions." (135) L'accident qui caractérise la trajectoire discontinue des phénomènes météorologiques s'articule au sein des passages consacrés explicitement à l'effet papillon dont tient compte la théorie du chaos. Les partisans d'un chaos déterministe expliquent le fameux effet à partir de l'identification d'un attracteur étrange indiquant que "l'atmosphère est un système dissipatif sensible aux conditions initiales" 31 . Au moment où le protagoniste découvre les origines véritables de sa mère, le discours a recours au métatexte afin d'exposer la courbe du temps: "Selon une théorie récente, un battement d'aile de papillon en Chine peut entraîner une catastrophe écologique en Amérique. C'est exactement ce que j'ai pensé : un battement d'aile me parvenait à travers le temps" (191), observe un Santerre bien au fait des développements scientifiques de son époque. Ici, le texte se réfère aux conclusions de Lorenz concernant les mouvements atmosphériques qui sont extrêmement sensibles aux conditions initiales. Mais l'image de l'attracteur étrange ne vaut pas seulement pour évoquer les turbulences psychologiques. Les segments narratifs ayant trait à la détérioration de l'état clinique du protagoniste épousent également un mouvement erratique, non conforme à la série causale. Soulignons que c'est Gaston Therroux, un médecin n'exerçant plus, qui a dépisté les signes avant-coureurs de la maladie: "J'ai peur pour toi. Regarde-toi. Tu es malade. Peutêtre plus qu'on penserait. Je n'aime pas ça. Fais vérifier ta formule sanguine au plus sacrant." (213) Le discours direct se fait d'ailleurs insistant: "Tu as mauvaise mine, Santerre. Un teint cendreux. Tu as maigri. As-tu fais faire des analyses?" (219) Une maladie que le personnage "n'a pu prévoir" a produit des effets irréversibles. Au demeurant, les soupçons du chasseur sont confirmés dans la mesure où le romancier est hospitalisé dès son retour en ville.

Comme le crabe qui marche de côté, Luc-Azade Santerre adopte une démarche scripturale dont la trajectoire n'est pas rectiligne, mais plutôt spiralée, à l'image d'un motif fractal. Fractal, faut-il le préciser, vient du latin fractus qui signifie irrégulier. Les structures fractales renvoient au principe d'auto-ressemblance postulé par les défenseurs de la théorie du chaos. Par le biais de la géométrie fractale, on peut cerner les formes com-

31. Ch. Ruhla, La physique du hasard. De Blaise Pascal à Niels Bohr, Paris, Hachette, 1988, p. 172. 
pliquées que crée la turbulence dans un espace ordinaire. Nombreuses sont les images du roman qui évoquent cette idée d'irrégularité révélatrice d'un espace-temps à plusieurs dimensions. Dès le premier chapitre, la voix masculine a recours au procédé de la réduplication pour endiguer une imagination débridée qui projette des simulacres de Sarah à travers la ville: "J'ai compris que je voyais des Sarah partout. Accoudées à un bar, assises à une terrasse. Des copies de Sarah partout. " (29) Comme nous l'avons signalé, les femmes diagonales rattachées au passé, la démarche latérale du crabe, la trajectoire oblique de l'éclair sont autant de syntagmes qui renvoient au processus mémoriel sollicité par l'écriture. Santerre affirme "avoir suivi les méandres d'une mémoire fractale" (186), alors qu'il décrit son périple en bordure du fleuve. Les métaphores fractales demeurent significatives dans un texte dont le jeu des frontières entre la réalité et la fiction brouille les traces de l'énonciation: "Pour une raison quelconque, je n'avais jamais réussi à vivre complètement dans le présent" (101), confie le narrateur.

Dans la perspective de lire le passé et de se situer par rapport aux générations précédentes, l'ancien dentiste quitte un milieu urbain occultant tout contact avec l'atavisme, la nature. Symbole de l'impossible identité à l'image d'un atome dénaturé, la ville est désertée par le héros qui ne voit en elle que le présent se renouvelant sans cesse à un rythme effréné. Celui-ci cherche à faire revivre le passé afin de déterminer comment les êtres, ou pour mieux dire les ancêtres, ont pu laisser des traces encore présentes, susceptibles d'influencer le moi: "Nous sommes le produit de ces marques qui parlent en silence, passent à travers nous" (215), conclut Santerre au terme de son expédition dans la forêt. Force est de constater le reflet du processus de création littéraire mis en abyme au sein du déplacement tourbillonnaire du personnage, épousant le relief du Saint-Laurent. L'imagination de Santerre se situe dans un processus d'écriture corrélé à un modèle dynamique: "À chaque seconde une image nouvelle émergeait de la précédente, toujours la même et chaque fois différente." (103) Fasciné par un hier qui modifie l'aujourd'hui, le narrateur se réfère lui-même à la géométrie de la nature développée par Benoit Mandelbrot ${ }^{32}$ : "Une fractale est un être

32. Pour une étude de la géométrie fractale, on peut consulter le livre de Benoit Mandelbrot: Les objets fractals. Forme, hasard et dimension. $3^{\mathrm{e}}$ édition révisée 
géométrique de forme découpée et ramifiée. Si l'on agrandit un détail du motif, on retrouve le motif tout entier." (187-188) Signalons que Mandelbrot a conçu une géométrie fractale pour étudier des objets naturels très divers dont le dénominateur commun consiste à se présenter comme des formes extrêmement irrégulières et ininterrompues. Pour engendrer l'irrégularité fractale, on met l'accent sur des constructions que domine le hasard. Les images qui en sont le caractère sont séduisantes; certaines sont des contrefaçons des montagnes, des nuages, des couchers de soleil, etc. Conformément au motif fractal dont la densité décroît à mesure que l'échelle s'agrandit, le trajet du personnage dans un espace scriptural transcendantal évoque la réduplication du même se métamorphosant au gré du temps vécu. Plus encore, les constellations identitaires rappellent la monade de Leibniz. Or, les signes sont des atomes. Chaque signe possède une signification qui se cristallise dans la fixité de l'expression, de la phrase. La construction d'une phrase sous-entend un processus de génération et de corruption sémiotique. En supposant que la phrase ait un sens, il faut admettre qu'il est transcendantal. D'ailleurs, un même énoncé ne peut être réitéré étant donné les situations d'énonciation toujours variables et capricieuses, comme la température. À la lumière de ces considérations, la phrase demeure comparable au fleuve d'Héraclite. À l'image d'un corps matériel soumis au changement, le signifiant du texte dérive d'un sens univoque.

Interprète incomparable en matière de signes renvoyant à des phénomènes d'ordre physique, Gaston Therroux contribue directement au projet romanesque de Santerre. En vertu des renseignements qu'il possède, l'homme des bois identifie les éléments déterminants de la préhistoire de Michelle et de Sarah Roche. En ce sens, le récit de Therroux constitue un élément essentiel au texte du je agonisant. Sous la forme du discours rapporté, l'ermite fait part à son visiteur de l'histoire d'Adrien Roche, le père de Michelle. Il s'agit d'un arpenteur. À bien des égards, celui-ci incarne le mythe d'un univers totalement déterministe, devenu l'idéologie de la domination de la nature. Suivant les renseignements biographiques recueillis, Adrien

et augmentée, Paris, Flammarion, 1989. Rappelons que la géométrie fractale s'oppose en principe à l'iconoclasme. Dynamique, le modèle fractal est défini comme un langage topologique et descriptif de la nature. 
Roche aurait tenté durant toute son existence de maîtriser les forces de la nature qui avaient démoli le rêve de son père. Ce dernier souhaitait faire fortune en exploitant une chute pour produire de l'électricité. Mais le barrage n'avait pas résisté aux pluies diluviennes. De père en fils s'est transmis un sentiment d'hostilité envers l'espace naturel: "La forêt et son désordre étaient [des] adversaires intimes." (210) Poussé par le ressentiment, le père de Michelle a converti un secteur de la forêt en une propriété privée, "[ayant] utilisé ses relations avec le parti au pouvoir pour changer le nom indien d'un lac et le rebaptiser du prénom de sa fille" (205). Or, le "lac Michelle" est un lieu discursif signifiant dans l'itinéraire romanesque de Santerre. L'existence du lac parvient à donner un sens à "l'éclair ancien" (128), à "l'effroi primitif" (129), ce fragment que le héros peut lire sur le visage de Michelle. Selon l'interprétation du sujet scripteur, il s'agit précisément de la "trace méconnaissable du rêve détruit de son grand-père" (215). Si l'on transpose en termes nietzschéens les spéculations du je narrant ayant trait à l'atavisme, on observe que l'"éclair ancien" dont il est question pourrait se rapporter à "la force [sic] des connaissances [qui] ne réside pas dans leur degré de vérité, mais dans leur ancienneté, dans leur degré d'assimilation, dans leur caractère de condition de vie "33. L'ancien dentiste apprend également que Roche rivalisait avec la tribu des Attikameks pour conserver un territoire dont les bornes étaient adjacentes à l'espace où vivaient les Amérindiens. Or, la réserve dont il est question est celle d'où est issue la mère amérindienne de Marc Martin, le père de Sarah. À partir de ces nouvelles informations, le narrateur parvient à interpréter des événements de longue date. Il se remémore surtout la peur irrépressible de Michelle en 1967, lorsqu'elle s'était rendue, en compagnie de Martin et de Santerre, à proximité des lieux où l'on avait retrouvé le corps sans vie de son père. La jeune femme n'avait pas supporté la présence de la forêt qu'elle avait qualifiée alors de "lugubre" (131), vocable dont le romancier se souvient avec précision. Enceinte, "Michelle avait compris ce qui se rencontrait fatalement dans son ventre." (207) À l'opposé des positions anticléricales et libérales dont se réclame Marc Martin, celles d'Adrien Roche représentent les valeurs traditionnelles d'un monde

33. Friedrich Nietzsche, Le gai savoir. "La gaya scienza". Fragments posthumes (Été 1881- été 1882). Édition revue et augmentée par Marc B. De Launay, Paris, Gallimard, 1982, p. 139. 
144

anachronique aux visées ultramontaines et nationalistes. L'arpenteur est l'auteur de nombreux traités dont certains ont trait au jansénisme. Prônant la croyance en l'action d'une fatalité divine, Roche s'est penché sur la doctrine de la prédestination au sein de ses "élucubrations jansénistes" (212). Ainsi que l'a vérifié Santerre, les textes postulent "que notre existence sur terre est provisoire et déchue, qu'il faut racheter une faute commise avant nous" (212). Tout bien considéré, Sarah se présente comme le fleuve d'Héraclite qui dissout les contraires: elle incarne à la fois l'héritage amérindien et celui du grand-père maternel asservi à des convictions religieuses orthodoxes.

Personnage issu de la postmodernité, Sarah s'accommode de la topologie accidentée du passé déterminant son parcours. Livrée à un hasard qui projette des individus atomisés de façon aléatoire dans l'Univers, l'existence comporte des traces à partir desquelles convergent des hypothèses et s'érigent des signes. En ce sens, la forêt constitue le point spatial où les ancêtres de Sarah se sont croisés: le grand-père maternel arpenteur et la grandmère amérindienne deviennent alors "d'autres atomes dotés de mémoire qui [échappent] à l'imagination mais [s'agglomèrent], engendrant dans le désordre et la confusion, l'écume du futur." (218) Autrement dit, le personnage de Sarah et sa généalogie servent à illustrer comment l'existence surgit du hasard, du clinamen qui introduit un temps nouveau, c'est-à-dire celui de l'écrit, de la mémoire. Le testament romanesque d'un homme ayant bifurqué de sa trajectoire culturelle et conditionnée de citadin indique comment "les choses et les mots [...] par la déclinaison échappent, pour le temps de leur existence, au flux irréversible de la dissolution [...] et le code échappe à l'entropie le temps du souvenir " 34 .

Le fondement de la connaissance est mis en question à travers les réflexions d'un personnage engagé dans une création littéraire. De fait, le je narrant tente de percer le sens de la trace généalogique: "On n'atteint jamais la fusion. La vérité nous est donnée à l'état de trace." (161) À la vue de la maison où il a séjourné avec sa mère durant les vacances d'été bien des années auparavant, Santerre comprend que ces lieux lui sont devenus étrangers en dépit de la "parcelle du passé conservée dans le

34. Michel Serres, op. cit., p. 185. 
présent [...]» (192). Ce filon concerne l'archéologie de la trace généalogique amenant le sujet à se percevoir à la lumière des gènes archaïques ou des animalcules cancérigènes qui dévorent son corps et sa pensée. Posée comme l'indice d'un signe établissant un pont entre les générations, la réduplication fractale de la trace revêt une importance capitale pour le personnage. Au fil de l'écriture, celui-ci cherche à saisir la signification de l'imprésentable, c'est-à-dire les marques indélébiles et naturelles d'un passé invisible. À partir du contenu diégétique qu'elle met en discours, la pratique autoréférentielle projette sur le mode analogique une identité permanente, logique et quasi numérique, d'une part, puis singulière et mouvante, d'autre part. À certains égards, cette dernière se rapporte dans le récit spéculaire à la différence derridéenne liant le processus de l'écriture à "une errance qu'il est impossible de référer à aucune origine; fragmentaire, morcelé, répété et réinscrit à l'infini de manière abyssale, ce tissu de traces toujours effaçables excède tout bon vouloir-dire [...]„35. Dans les manuscrits d'un sujet qui se meurt, la trace comme vecteur identitaire renvoie à ce qui est imprésentable, et elle traduit la différence qui modifie la substance du même. En cela, la trace de l'écrit se distingue d'une logique binaire, du représentable. C'est donc la part d'imprésentable dans le représentable, de discontinu dans le continu, qui imprègne l'écriture de Santerre.

Le retour à la philosophie des atomistes de l'Antiquité grecque et latine dans $L a$ démarche du crabe aura certes permis de mieux cerner la position postmoderne et relativiste de la romancière Monique LaRue. Au sein de la fiction, la sphère mouvante des apparences n'entre pas dans un rapport d'opposition stricte avec le monde des idées et des essences immatérielles. La dimension spéculaire des scénarios contenus dans l'autobiographie fictive de Luc-Azade Santerre situe l'identité du personnage entre le signifiant et le signifié, la forme et la signification, le dur et le mou. C'est une logique de l'entre-deuxmondes qui grave le récit au sein duquel le procédé de la mise

35. Jacques Derrida et Pierre-Jean Labarrière, Altérités, Paris, Osiris, 1986, p. 23. 
en abyme produit un effet d'autoreprésentation onirique: "Je touchais, je soupesais la part de moi-même qui était l'œuvre [de ma mère]: Dr Luc-Azade Santerre. Chirurgien dentiste. Du solide. Mais le vide en dessous me fascinait beaucoup plus." (218) Or, l'esthétique de l'imprésentable livrée à travers les phénomènes aléatoires issus de la géométrie du chaos s'inscrit dans le courant d'une pensée postmoderne. Sise au cour des apparences, la réduplication fractale dont s'inspire le sujet agonisant pour produire sa création littéraire édulcore le principe d'une identité unitaire. Au lieu de procéder de la raison souveraine valorisée par la logique classique, le récit fictif pénètre les couches de signification d'un "réel voilé et incertain". En vertu de mécanismes de structuration qui se fondent sur le hasard et les lois inextricables de la nature, le réel appréhendé par la fiction dédoublée ne peut être reconstitué suivant l'ordre de la causalité stricte. Bien au contraire, les événements qui façonnent le disours romanesque prennent place dans un processus mémoriel dont la trajectoire discontinue est apparentée au mouvement tourbillonnaire des atomes décrit par les Anciens. Bref, l'écriture en tant que lieu du souvenir et de l'imagination, élude les contradictions identitaires dont elle magnifie les traces. 